\title{
Levetiracetam in Neonatal seizures
}

\author{
Manish Swami ${ }^{1}$ and Jaya Shankar Kaushik ${ }^{2}$ \\ From Departments of Pediatrics, ${ }^{1}$ All India Institute of Medical Sciences, Delhi, and ${ }^{2}$ Pandit Bhagwat Dayal Sharma Postgraduate \\ Institute of Medical Sciences, Rohtak, Haryana; India. ${ }^{2}$ jayashankarkaushik@gmail.com
}

$\mathrm{N}$ eonatal seizures are one of the most commonly encountered emergencies in Pediatrics. About two-thirds of cases of neonatal seizures are due to Hypoxic ischemic encephalopathy (HIE), which affects approximately 1-2/1000 live births [1]. Phenobarbitone and phenytoin are the commonly used anticonvulsants for neonatal seizures after correction of hypoglycemia and hypocalcemia. Good central nervous system (CNS) penetration, low protein binding, and optimal efficacy make phenobarbitone the drug of choice for the management of neonatal seizures [2]. However, longterm use of phenobarbitone is associated with an impairment of maturation of synapses and enhanced neuronal apoptosis.

Levetiracetam is a pyrrolidine derivative that binds to the synaptic vesicle protein SV2a. Some of the favorable pharmacokinetics of Levetiracetam include minimal protein binding, safety in liver failure, minimal adverse effect profile, and nearly no drug interactions. Levetiracetam is approved for use by the Central Drug Standard Control Organization (CDSCO) and Food and Drug Administration (FDA) as an adjunctive therapy for partial onset seizure in infants beyond one month of age. However, the drug has been used off-label in the management of neonatal seizures [3].

In this issue of Indian Pediatrics, Gowda, et al. [4] provide robust evidence to support the use of levetiracetam in neonatal seizures. In their study, 43 out of 50 neonates who received $20 \mathrm{mg} / \mathrm{kg}$ of levetiracetam achieved cessation of seizure with no recurrence in the subsequent 24 hours. Moreover, 16 of the 19 babies who failed to respond to two doses of phenobarbitone (cumulative dose of $30 \mathrm{mg} / \mathrm{kg}$ ) also responded to single dose of $20 \mathrm{mg} / \mathrm{kg}$ of levetiracetam. This raises curiosity to know the baseline characteristics of babies who responded remarkably to levetiracetam. The majority of the neonates in this study included term neonates with a birth weight of 2.5-2.7 kg, enrolled at a mean age of 8-9 days. Neonatal seizures secondary to HIE mostly present within first 24 hours, and those secondary to neonatal cerebrovascular stroke occur within the first 72 hours of birth. Hence, the study results of superior efficacy of levetiracetam over phenobarbitone in neonatal seizures need to be interpreted with caution keeping in mind these baseline characteristics.

There is a considerable variation in dosing of levetiracetam for neonatal seizures with dose as high as $50-60 \mathrm{mg} / \mathrm{kg}[5,6]$. Gowda, et al. [4] have demonstrated superior efficacy with a dose of $20 \mathrm{mg} / \mathrm{kg}$ of levetiracetam. There are limited studies on the pharmacokinetics of levetiracetam in the neonatal age group. One such research has demonstrated that levetiracetam has a longer half-life and more extensive volume of distribution in neonates when compared to older children and adults [7]. We eagerly look forward to the results of a phase II trial planned to determine the optimal dosing of levetiracetam as the first-line drug for the management of neonatal seizures in HIE [8].

Levetiracetam in the rat model has demonstrated to lack any neurotoxic effects at all therapeutic doses [9]. Moreover, it is also known to decrease the number of apoptotic cells in the hippocampus and cerebral cortex when administered to babies with hypoxia [10]. Although the study by Gowda, et al. [4] supports the use of levetiracetam in neonatal seizures, future trials could be planned to target a more focused homogenous population like term neonates with HIE. Also, the proposed research must ideally match standard recommendations for designing a clinical trial on neonatal seizures to allow replicability and comparability of study findings [11]. There is a significant emphasis on the quantification of seizure occurrence with continuous EEG monitoring being mandatory to assess the treatment outcome in trials on neonatal seizures.

At the outset, everything looks good about levetiracetam: favorable pharmacokinetics, good efficacy, and lack of any significant adverse events. However, currently the evidence is insufficient to recommend its 
routine use in neonatal seizures, especially those due to HIE. In coming years, levetiracetam will be a promising alternative to phenobarbitone in the management of neonatal seizures.

Funding: None; Competing interests: None stated.

\section{REFERENCES}

1. Saliba RM, Annegers JF, Walker DK, Tyson JE, Mizrahi EM. Incidence of neonatal seizures in Harris County, Texas, 1992-1994. Am J Epidemiol. 1999;150:763.

2. El-dib M, Soul JS. The use of Phenobarbital and other anti-seizure drugs in the newborn. Semin Fetal Neonatal Med. 2017;22:321-7.

3. Silverstein FS, Ferriero DM. Off-label use of antiepileptic drugs for the treatment of neonatal seizures. Pediatr Neurol. 2008;39:77-9.

4. Gowda VK, Romana A, Shivanna NH, Benakappa N, Benakappa A. Levetiracetam versus phenobarbitone in neonatal seizures - A randomized controlled trial. Indian Pediatr. 2019;56:643-6.

5. Khan O, Chang E, Cipriani C, Wright C, Crisp E, Kirmani B. Use of intravenous levetiracetam for management of acute seizures in neonates. Pediatr Neurol. 2011;44:265-9.
6. Shoemaker MT, Rotenberg JS. Levetiracetam for the treatment of neonatal seizures. J Child Neurol. 2007;22: 95-8.

7. Merhar SL, Schibler KR, Sherwin CM, Meinzen-Derr J, Shi J, Balmakund $\mathrm{T}$, et al. Pharmacokinetics of levetiracetam in neonates with seizures. J Pediatr. 2011;159:152-4.e3.

8. Favrais G, Ursino M, Mouchel C, Boivin E, Jullien V, Zohar S, et al. Levetiracetam optimal dose-finding as firstline treatment for neonatal seizures occurring in the context of hypoxic-ischaemic encephalopathy (LEVNEONAT-1): study protocol of a phase II trial. BMJ Open. 2019;9:e022739.

9. Manthey D, Asimiadou S, Stefovska V, Kaindl AM, Fassbender J, Ikonomidou C, et al. Sulthiame but not levetiracetam exerts neurotoxic effect in the developing rat brain. Exp Neurol. 2005;193:497-503.

10. Kilicdag H, Daglioglu K, Erdogan S, Guzel A, Sencar L, Polat $\mathrm{S}$, et al. The effect of levetiracetam on neuronal apoptosis in neonatal rat model of hypoxic ischemic brain injury. Early Hum Dev. 2013;89:355-60.

11. Stevenson NJ, Vanhatalo S. Designing a trial for neonatal seizure treatment. Semin Fetal Neonatal Med. 2018;23:213-7. 